MATHEMATICS OF COMPUTATION

Volume 70, Number 233, Pages 107-119

S $0025-5718(00) 01270-9$

Article electronically published on May 23, 2000

\title{
WEAKENED ACUTE TYPE CONDITION FOR TETRAHEDRAL TRIANGULATIONS AND THE DISCRETE MAXIMUM PRINCIPLE
}

\author{
SERGEY KOROTOV, MICHAL KŘÍŽEK, AND PEKKA NEITTAANMÄKI
}

\begin{abstract}
We prove that a discrete maximum principle holds for continuous piecewise linear finite element approximations for the Poisson equation with the Dirichlet boundary condition also under a condition of the existence of some obtuse internal angles between faces of terahedra of triangulations of a given space domain. This result represents a weakened form of the acute type condition for the three-dimensional case.
\end{abstract}

\section{Introduction: MAXIMUM PRINCIPLE}

In this paper we prove the validity of a discrete maximum principle for continuous piecewise linear finite element approximations for the Poisson equation with the Dirichlet boundary condition

$$
\begin{aligned}
& -\Delta u=f \quad \text { in } \quad \Omega \text {, } \\
& u=g \quad \text { on } \quad \partial \Omega \text {, }
\end{aligned}
$$

where $f \in L^{2}(\Omega), g \in C(\partial \Omega)$ and $\Omega \subset \mathbb{R}^{3}$ is a bounded domain with a Lipschitzcontinuous polyhedral boundary $\partial \Omega$.

First we formulate the strong maximum principle for the continuous problem (cf. 14]) as follows.

A linear partial differential operator of the second order $\mathcal{L}$ defined on a space of suitably smooth functions, which are in turn defined on a bounded domain $\Omega \subset \mathbb{R}^{d}, d \in\{1,2, \ldots\}$, with a boundary $\partial \Omega$, is said to satisfy the strong maximum principle if

$$
\mathcal{L} u(x) \leq 0 \quad \forall x \in \Omega \quad \text { and } \quad u(s) \leq 0 \quad \forall s \in \partial \Omega
$$

imply that

$$
u(x) \leq 0 \quad \forall x \in \bar{\Omega} .
$$

Received by the editor January 26, 1999.

2000 Mathematics Subject Classification. Primary 65N30.

Key words and phrases. Maximum principle, Poisson equation, weakened acute type condition, linear tetrahedral finite element.

The first author was partly supported by the Academy of Finland, Grant no. 752205, and partly by the Mittag-Leffler Institute, Djursholm, Sweden.

The second author was supported by the Grant no. 201/98/0528 of the Grant Agency of Czech Republic. 


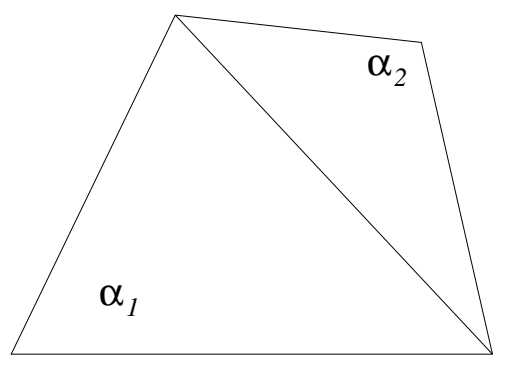

a)

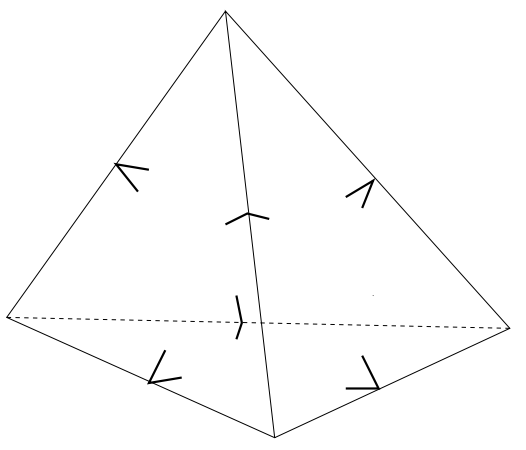

b)

FIGURE 1.

If $\mathcal{L}$ satisfies the condition $\mathcal{L} v \equiv 0$ for any constant function $v$ and if $\mathcal{L} u(x) \leq 0$ for all $x \in \Omega$, then the following maximum principle holds (cf. [6. p. 31])

$$
\max _{x \in \bar{\Omega}} u(x)=\max _{s \in \partial \Omega} u(s)
$$

Note that this is precisely the case of our problem (1)-(2).

It is natural to ask whether the corresponding discrete problem satisfies the same principle as the continuous problem. This question for linear elliptic equations is considered in 4]: in the two-dimensional case the discrete maximum principle is proved for continuous piecewise linear finite element approximations if all angles $\alpha$ in the triangulation are not greater than $\frac{\pi}{2}$ (the so-called acute type condition). However, it is noted that the discrete maximum principle holds for continuous piecewise linear finite element approximations for our problem under the following weaker condition ([17, p. 78]): for every pair $\left(\alpha_{1}, \alpha_{2}\right)$ of angles opposite a common edge of some given pair of adjacent triangles of the triangulation we have $\alpha_{1}+\alpha_{2} \leq$ $\pi$, (see Figure 1a). In [13], it is shown that the discrete maximum principle may still hold in some cases if both angles in such a pair are greater than $\frac{\pi}{2}$.

In the three-dimensional case this problem is studied in [10, where the authors prove the validity of the discrete maximum principle under the condition that all internal angles between faces of all tetrahedra in the triangulation of $\bar{\Omega}$ are not greater than $\frac{\pi}{2}$ (a natural generalization of the acute type condition to the threedimensional case, see Figure 1b). Moreover, they prove the result for a nonlinear elliptic equation taking into account the effect of numerical integration (cf. also [5] for the two-dimensional case).

If an edge is surrounded by four tetrahedra, then all four associated angles have to be equal to $\frac{\pi}{2}$ to satisfy the acute type condition introduced in [10]. This is a quite restrictive property, which is difficult to satisfy especially when performing refinements by "midlines in $3 d$ "; see the four "internal" tetrahedra in Figure 2 and their common edge (cf. [9]). Some special examples of acute type triangulations of polyhedra are given in [10]. They are based on Delaunay triangulations (cf. [7]).

In this paper we consider problem (1)-(2) and show that the above-mentioned acute type condition can be weakened and some obtuse internal angles between some faces of tetrahedra can be allowed under certain conditions on the triangulation of 


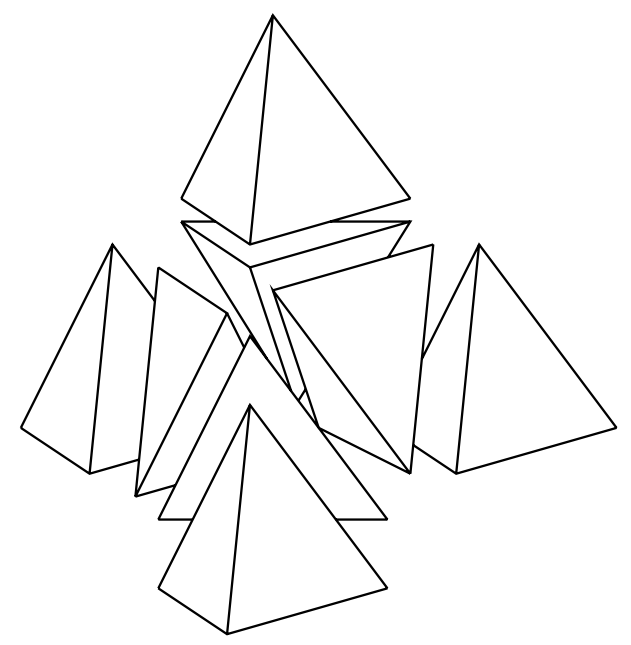

FIGURE 2.

a given domain and the discrete maximum principle still holds. An example of such a triangulation is given in Section 4 .

Other papers devoted to discrete maximum principles include, e.g., [1, 8], [15], [16].

\section{THE DISCRETE PROBLEM}

If we examine proofs of discrete maximum principles to hold, then we see that they are all based on the following fact: the corresponding discrete problems are of nonnegative type (cf. 4]). This means that the finite element approximate problem leads to the solution of a system of linear equations of the form

$$
\bar{A} \bar{u}=\bar{b},
$$

where the matrix $\bar{A}=\left(a_{i j}\right)_{i, j=1}^{\bar{n}}$, besides being nonsingular, satisfies the following conditions:

$$
\begin{aligned}
a_{i i} & >0, \quad i=1, \ldots, \bar{n} \\
\sum_{j=1}^{\bar{n}} a_{i j} & \geq 0, \quad i=1, \ldots, \bar{n} \\
a_{i j} & \leq 0, \quad i, j=1, \ldots, \bar{n}, \quad i \neq j .
\end{aligned}
$$

Here $\bar{u}=\left(u_{1}, \ldots, u_{\bar{n}}\right)$ and for the Dirichlet boundary problem the components $u_{n+1}, \ldots, u_{\bar{n}}$ are given by values of $g$ at nodes belonging to the boundary $\partial \Omega$.

Remark 1. If (6) holds, then the matrix $\bar{A}$ is known to have a nonnegative inverse (see [18]), which implies the validity of the discrete maximum principle (cf. [10]).

The conditions on $\bar{A}$ can be still reduced:

Theorem 1. Let the finite element matrix $\bar{A}$ of the problem (1)-(2) be monotone (i.e., nonsingular with a nonnegative inverse) and let (6b) hold. Then whenever 
$f \leq 0$, the following discrete maximum principle is valid:

$$
\max _{1 \leq i \leq \bar{n}} u_{i}=\max _{n+1 \leq j \leq \bar{n}} u_{j}
$$

The proof can be found in [3, p. 342].

Remark 2. In our paper we will allow some obtuse angles in the triangulation (which may cause condition (6c) to not hold), such that the matrix $\bar{A}$ remains monotone, and, therefore, in view of Theorem 1 , the discrete maximum principle holds under weaker conditions than in 10 .

Further we give some notation and conditions on the triangulations used. By $K$ (possibly with a subscript) we always mean a closed tetrahedron. The symbol $\mathcal{T}_{h}$ denotes a triangulation of $\bar{\Omega}$ into tetrahedra, whose nodes are $B_{1}, \ldots, B_{\bar{n}}$. We denote by $\phi_{1}, \ldots, \phi_{\bar{n}}$ continuous and piecewise linear basis functions defined in a standard way, i.e., $\phi_{i}\left(B_{j}\right)=\delta_{i j}$ for $i, j=1, \ldots, \bar{n}$, where $\delta_{i j}$ is the Kronecker symbol. We also assume that $\left\{\mathcal{T}_{h}\right\}_{h \rightarrow 0}$ is a strongly regular family of triangulations, i.e., there exists a positive constant $C_{3}^{\prime}$ independent of $h$ such that

$$
C_{3}^{\prime} h^{3} \leq \operatorname{meas}_{3} K \quad \forall h \in\left(0, h_{0}\right) \quad \forall K \in \mathcal{T}_{h},
$$

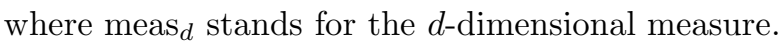

Let $B_{1}, \ldots, B_{n}$ be nodes that do not lie on $\partial \Omega$ and let $m$ be the number of nodes lying on $\partial \Omega$, i.e., $\bar{n}=n+m$.

Let $a(\cdot, \cdot)$ be a bilinear form associated with the problem (1)-(2), i.e.,

$$
a(u, v)=\int_{\Omega} \nabla u \cdot \nabla v d x .
$$

We define the basic finite element matrix $A$ to be the $n \times n$ matrix whose entries are

$$
a_{i j}=a\left(\phi_{i}, \phi_{j}\right), \quad i, j=1, \ldots, n,
$$

and the $n \times m$ boundary matrix $Z$ with entries $z_{i j}$ given as

$$
z_{i j}=a\left(\phi_{i}, \phi_{n+j}\right), i=1, \ldots, n, \quad j=1, \ldots, m .
$$

We also set

$$
b_{i}=\int_{\Omega} f \phi_{i} d x, i=1, \ldots, n .
$$

What we actually need is to solve the problem

$$
A u=b-Z g,
$$

where $g=\left(g\left(B_{n+1}\right), \ldots, g\left(B_{\bar{n}}\right)\right)^{T}=\left(g_{1}, \ldots, g_{m}\right)^{T}$. However, to simplify the proof of the main result of Section 3, it is more convenient to consider an extended form of this system, namely, the $\bar{n} \times \bar{n}$ linear system of equations (like (5)), where $\bar{b}=\left(b_{1}, \ldots, b_{n}, g_{1}, \ldots, g_{m}\right)^{T}$ and $\bar{A}$ is the $\bar{n} \times \bar{n}$ matrix having the form

$$
\bar{A}=\left[\begin{array}{cc}
A & Z \\
0 & I
\end{array}\right],
$$

where $I$ is the identity matrix.

Further we analyze of the structure of entries of $\bar{A}$. First note that if two different vertices $B_{i}$ and $B_{j}$ have no common edge, then

$$
\text { meas }_{3}\left(\operatorname{supp} \phi_{i} \cap \operatorname{supp} \phi_{j}\right)=0, \quad \text { i.e., } \quad a_{i j}=0 .
$$




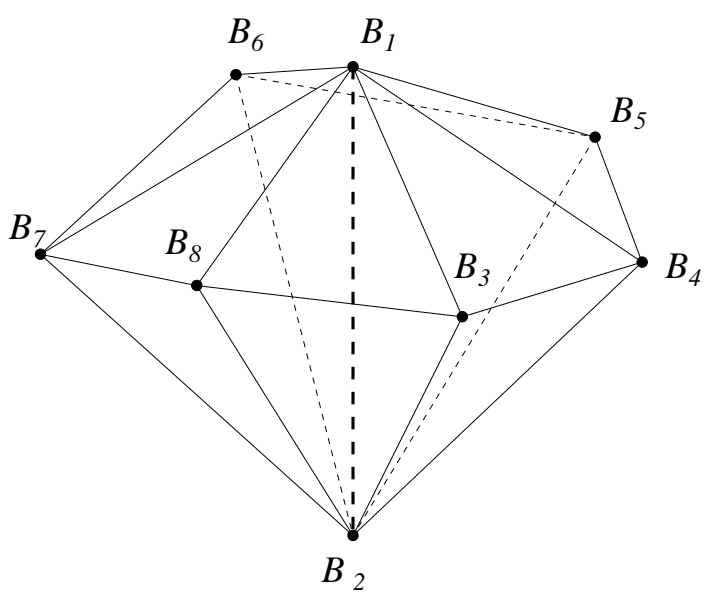

FiguRE 3.

Let us now consider an edge denoted by $B_{1} B_{2}$ for simplicity, and let $B_{3}, B_{4}, \ldots$, $B_{M+2}$ be another set of vertices in an appropriate order, which are connected with edges to both $B_{1}$ and $B_{2}$. Then we observe that

$$
\operatorname{supp} \phi_{1} \cap \operatorname{supp} \phi_{2}=\bigcup_{r=1}^{M} K_{r},
$$

where the symbol $K_{r}$ denotes a tetrahedron $B_{1} B_{2} B_{r+2} B_{r+3}$ (see Figure 3 with $M=6)$ and we define $B_{M+3} \equiv B_{3}$. We see that the tetrahedra $K_{1}, \ldots, K_{M}$ are "surrounding" the edge $B_{1} B_{2}$.

From (9)-(10) we particularly have that

$$
a_{12}=\left.\left.\sum_{r=1}^{M} \int_{K_{r}} \nabla \phi_{1}\right|_{K_{r}} \nabla \phi_{2}\right|_{K_{r}} d x,
$$

where $\left.\phi_{j}\right|_{K_{r}} \in P_{1}\left(K_{r}\right), j=1,2, r=1, \ldots, M$, and $P_{1}\left(K_{r}\right)$ denotes the space of linear polynomials over $K_{r}$.

In what follows we always use the notation $\alpha_{s t}^{K}$ for the internal angle in $K$ (between faces), which is opposite to the edge $B_{s} B_{t}$, and the notation $S_{i j k}$ for face of $K$ with vertices $B_{i}, B_{j}, B_{k}$.

Lemma 1 . Let $K$ be an arbitrary tetrahedron with vertices $B_{1}, B_{2}, B_{3}, B_{4}$ and let $p, q \in P_{1}(K)$ be such that

$$
\begin{array}{lll}
p\left(B_{1}\right)=1, p\left(B_{i}\right)=0 & \text { for } & i \neq 1, \\
q\left(B_{2}\right)=1, q\left(B_{j}\right)=0 & \text { for } & j \neq 2,
\end{array}
$$

then

$$
\begin{gathered}
\|\nabla p\|=\frac{\operatorname{meas}_{2} S_{234}}{3 \operatorname{meas}_{3} K}, \\
\nabla p \cdot \nabla q=-\frac{\operatorname{meas}_{2} S_{234} \cdot \operatorname{meas}_{2} S_{134}}{9\left(\operatorname{meas}_{3} K\right)^{2}} \cos \alpha_{12}^{K} .
\end{gathered}
$$

For the proof see [10, Lemma 3.1]. 
Remark 3. From (14) it follows that the most undesirable case (in the notation of Figure 3 ) is when all angles between faces $S_{1, r+2, r+3}$ and $S_{2, r+2, r+3}, r=1, \ldots, M$, are not less than $\frac{\pi}{2}$ and at least one is greater than $\frac{\pi}{2}$. Then in view of (12) and (14) we observe that $a_{12}>0$, i.e., the condition (6c) does not hold for the corresponding matrix $\bar{A}$.

Note also that if not all such angles are obtuse and there are some acute ones we can still get a negative value (as well as a positive one) of $a_{12}$, since it is (cf. (12)) a sum of $M$ terms over $K_{r}, r=1, \ldots, M$.

In this paper we consider the situation (cf. Remark 2) when some positive offdiagonal elements in the matrix $\bar{A}$ may also appear. To prove that the matrix $\bar{A}$ is, under certain conditions, still monotone and therefore that the discrete maximum principle holds by Theorem 1, we use the Bramble-Hubbard decomposition theorem from [2].

Before giving a formulation of this theorem we introduce some further notation. We assume for the moment that $A$ is an arbitrary $n \times n$ matrix for which (6a) and (6b) are valid, and that there exists a nonempty set $J(A)$ of numbers of rows of $A$ such that for every $k \in J(A)$ we have $\sum_{j=1}^{n} a_{k j}>0$. Now for $i \notin J(A)$ we define a connection in $A$ from $i$ to $J(A)$ to be a finite sequence of nonzero elements of the form $a_{i j_{1}}, a_{j_{1} j_{2}}, a_{j_{2} j_{3}}, \ldots, a_{j_{s} k}$, where $k \in J(A)$, cf. [18]. We assume also that there exists at least one such a connection in $A$ for every $i \notin J(A)$.

From now on we assume that the matrix $B$, which we will be dealing with in the following theorem, is given by $B=(\operatorname{diag} \bar{A})^{-1} \bar{A}$, where $\operatorname{diag} \bar{A}$ denotes the diagonal matrix whose diagonal coincides with that of $\bar{A}$. Note that from a trivial observation $\sum_{j=1}^{\bar{n}} \phi_{j} \equiv 1$ in $\bar{\Omega},(9)$ and (10) we may easily derive that both $\bar{A}$ and $B$ satisfy $(6 \mathrm{a})$ and $(6 \mathrm{~b})$, and $J(\bar{A})=J(B)=\{n+1, \ldots, \bar{n}\}$.

Theorem 2. Let a matrix $B$ have a unit diagonal, satisfy (6b) and let $J(B) \neq \emptyset$. If there exists a splitting $B=I-C-D$, where

$$
\begin{gathered}
\operatorname{diag} C=0, \\
I-C \quad \text { satisfies (6b) and (6c), } \\
(I-C)^{-1} D \text { is nonnegative, } \\
\text { for each } i \notin J(B) \text { there exists a connection in } C \text { from } i \text { to } J(B),
\end{gathered}
$$

then $B$ is monotone, i.e., $B^{-1}$ exists and is nonnegative.

For the proof see [2] p. 352].

Remark 4. Actually, it will be difficult to check only condition (15c). To simplify this procedure we define the following decomposition of the matrix $D$ (note that it is different from that given in [13]):

$$
D=D^{\text {pos }}+D^{\text {neg }},
$$

where the entries of matrices $D^{\text {pos }}=\left(d_{i j}^{\text {pos }}\right)_{i, j=1}^{\bar{n}}$ and $D^{\text {neg }}=\left(d_{i j}^{\text {neg }}\right)_{i, j=1}^{\bar{n}}$ are defined in the following fashion. If an entry $d_{i j}$ consists of several contributions, i.e.,

$$
d_{i j}=\sum_{l=1}^{M_{i j}} d_{i j}^{l},
$$


then we set

$$
d_{i j}^{\mathrm{pos}}=\sum_{d_{i j}^{l} \geq 0} d_{i j}^{l} \quad \text { and } \quad d_{i j}^{\mathrm{neg}}=\sum_{d_{i j}^{l} \leq 0} d_{i j}^{l} .
$$

Further, from the trivial relation

$$
(I-C)^{-1} D=D^{\text {pos }}+(I-C)^{-1}\left(D^{\text {neg }}+C D^{\text {pos }}\right),
$$

we observe that in order to verify $(15 \mathrm{c})$ it is enough to prove that $D^{\text {neg }}+C D^{\text {pos }}$ is a nonnegative matrix, since both $D^{\text {pos }}$ and $(I-C)^{-1}$ are obviously nonnegative in view of (15a), (15b) and Remark 1.

Remark 5. Taking into account Theorems 1 and 2, we observe that if one finds a suitable splitting of $B=(\operatorname{diag} \bar{A})^{-1} \bar{A}$ satisfying $(15 \mathrm{a})-(15 \mathrm{~d})$, then the discrete maximum principle (7) still holds.

Let us define the entries of the matrices $C$ and $D$ as follows:

$$
\begin{aligned}
& c_{i j}=-a_{i j}^{\mathrm{neg}} / 2 a_{i i}, \\
& d_{i j}=-a_{i j}^{\text {neg }} / 2 a_{i i}-\left(1-\delta_{i j}\right) a_{i j}^{\mathrm{pos}} / a_{i i},
\end{aligned}
$$

where $i=1, \ldots, n, j=1, \ldots, \bar{n}$, and the other their entries are zeros.

\section{MAin RESUlt}

Before presenting the main result - Theorem 3-we make the following observations on the triangulations used.

Lemma 2. Let $\left\{\mathcal{T}_{h}\right\}$ be a strongly regular family of triangulations of $\bar{\Omega}$. Then there exist positive constants $C_{1}^{\prime}, C_{1}^{\prime \prime}, C_{2}^{\prime}, C_{2}^{\prime \prime}, C_{3}^{\prime}, C_{3}^{\prime \prime}, \theta_{0}$ and $h_{0}$ independent of $h$ such that for all $h \in\left(0, h_{0}\right)$, all $K \in \mathcal{T}_{h}$ and all their interior angles $\theta$, faces $S$ and edges $e$, we have

$$
\begin{aligned}
C_{1}^{\prime} h & \leq \text { meas }_{1} e \leq C_{1}^{\prime \prime} h, \\
C_{2}^{\prime} h^{2} & \leq \text { meas }_{2} S \leq C_{2}^{\prime \prime} h^{2}, \\
C_{3}^{\prime} h^{3} & \leq \operatorname{meas}_{3} K \leq C_{3}^{\prime \prime} h^{3}, \\
\theta_{0} & \leq \theta \leq \pi-\theta_{0} .
\end{aligned}
$$

The proof follows immediately from the property of the strong regularity (8).

Lemma 3. Let $\mathcal{F}=\left\{\mathcal{T}_{h}\right\}_{h \rightarrow 0}$ be a strongly regular family of triangulations of $\bar{\Omega}$. Then there exists a positive constant $\theta_{1}$ independent of $h$ such that if $K$ is an arbitrary tetrahedron from $\mathcal{T}_{h}$ and $\alpha_{1}, \alpha_{2}, \alpha_{3}$ are internal angles between an arbitrary face of $K$ and three remaining faces of $K$ adjacent to this face, then

$$
\min \left(\alpha_{1}, \alpha_{2}, \alpha_{3}\right) \leq \frac{\pi}{2}-\theta_{1} .
$$

Proof. Let $\mathcal{T}_{h} \in \mathcal{F}, K \in \mathcal{T}_{h}$ and a face $S$ of $K$ be arbitrary. Let $\rho$ be the radius of the inscribed circle $\mathcal{O}$ to $S$ and let $v$ be the length of the spatial altitude of $K$ onto the face $S$. If the attitude ends at the centre of $\mathcal{O}$, then $\alpha_{1}=\alpha_{2}=\alpha_{3}=\arctan \frac{v}{\rho}$; otherwise at least one of these angles is less than $\arctan \frac{v}{\rho}$.

From (18) and (19) we get $v \leq C_{1}^{\prime \prime} h$ and

$$
\rho=\frac{2 \operatorname{meas}_{2} S}{\operatorname{meas}_{1} \partial S} \geq \frac{2 C_{2}^{\prime} h^{2}}{3 C_{1}^{\prime \prime} h} .
$$


Hence, we find that

$$
\min \left(\alpha_{1}, \alpha_{2}, \alpha_{3}\right) \leq \arctan \frac{v}{\rho} \leq \arctan \frac{3\left(C_{1}^{\prime \prime}\right)^{2}}{2 C_{2}^{\prime}} .
$$

The last term is independent of $h$ and less than $\pi / 2$. From here the existence of some $\theta_{1}>0$ follows.

From now on we impose the following basic assumption on the triangulations used:

(BA) Let $a_{i j}>0$ for some $i, j \in\{1, \ldots, \bar{n}\}, i \neq j$. For simplicity assume that $i=1$ and $j=2$ in order to keep the notation of Figure 3. In such a case, we require that there exist vertices $B_{r}, r \in\{3, \ldots, M+2\}$, and two tetrahedra $K_{1, r}$ and $K_{2, r} \in \mathcal{T}_{h}$, having $B_{1} B_{r}$ and $B_{2} B_{r}$ as their edges, respectively, so that

$$
\alpha_{k, r}^{K, r} \leq \frac{\pi}{2}-\theta_{2}, \quad k=1,2,
$$

where $\theta_{2}$ is some positive constant independent of $h$.

Remark 6. Observe that we always have $\theta_{1} \leq \theta_{2}$. However, the numerical example of Section 4 shows that it can happen $\theta_{1}=\theta_{2}$ (cf. (31)).

Note that $M_{\max } \leq\left[\frac{2 \pi}{\theta_{0}}\right]$, where $M_{\max }$ is the maximum number of tetrahedra around the same edge and the constant $\theta_{0}$ is from (21).

Theorem 3. Let $\left\{\mathcal{T}_{h}\right\}$ be a strongly regular family of triangulations of $\bar{\Omega}$ leading to the matrix $\bar{A}$ given by (11) with entries satisfying (BA).

Let, in case $a_{i j}>0$, one of the following two conditions (in the notation of Figure $3 a_{i j} \equiv a_{12}$ ) hold:

1. None of the nodes $B_{3}, B_{4}, \ldots, B_{M+2}$ belongs to $\partial \Omega$;

2. The function $g$ is constant and $B_{2} B_{i} B_{i+1} \cap \partial \Omega=B_{2} B_{i} B_{i+1}$ or $\emptyset, i=$ $3,4, \ldots, M+2, B_{M+3} \equiv B_{3}$.

Then the discrete maximum principle (7) holds for continuous piecewise linear finite element approximations of the problem (1)-(2) if internal angles between faces of tetrahedra from $\mathcal{T}_{h}$ are not greater than $\frac{\pi}{2}+\varepsilon$, where

$$
\sin \varepsilon \leq \min \left(\frac{C_{2}^{\prime}}{2 C_{2}^{\prime \prime}} \sin \theta_{1}, \frac{81}{4 M_{\max }} \cdot \frac{\left(C_{2}^{\prime}\right)^{4}}{\left(C_{2}^{\prime \prime}\right)^{4}} \cdot \frac{\left(C_{3}^{\prime}\right)^{2}}{\left(C_{3}^{\prime \prime}\right)^{2}} \cdot \sin ^{2} \theta_{2}\right) .
$$

Proof. From now on we always assume that $B_{1} \notin \partial \Omega$, since the case $B_{1}, B_{2} \in \partial \Omega$ is trivial, see (11).

We check whether the assumptions of Theorem 2 are satisfied (cf. Remark 5).

First we consider the case when none of the other nodes $B_{2}, \ldots, B_{M+2}$ lies on $\partial \Omega$. Obviously, (15a) is valid in view of definition (17a) of the matrix $C$.

Further we prove that (15b) holds. Using again (17a) and the fact that matrix $B$ satisfies (6b), we observe that

$$
\begin{aligned}
1-\sum_{j=1}^{\bar{n}} c_{i j} & =1+\sum_{j \neq i} a_{i j}^{\mathrm{neg}} / 2 a_{i i} \\
& =\left[1+\sum_{j \neq i} a_{i j} / a_{i i}\right]+\left[\sum_{j \neq i}\left(a_{i j}^{\mathrm{neg}} / 2 a_{i i}-\left(a_{i j}^{\mathrm{neg}}+a_{i j}^{\mathrm{pos}}\right) / a_{i i}\right)\right] \\
& \geq\left[\sum_{j \neq i}\left(-a_{i j}^{\mathrm{neg}} / 2 a_{i i}-a_{i j}^{\mathrm{pos}} / a_{i i}\right)\right]=-1 / a_{i i} \sum_{j \neq i}\left(a_{i j}^{\mathrm{neg}} / 2+a_{i j}^{\mathrm{pos}}\right) .
\end{aligned}
$$




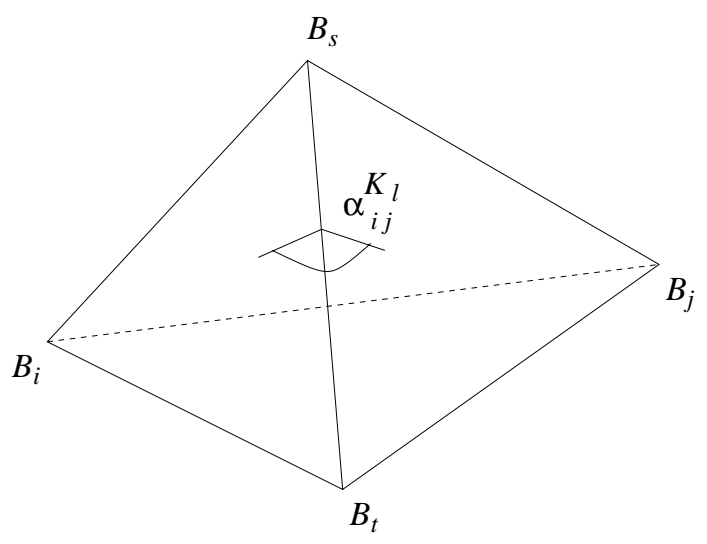

FIGURE 4.

Now we give a sufficient condition for the right-hand side to be nonnegative. Suppose that for some fixed $i$ and $j, i \neq j$, there exists a nonnegative contribution over some tetrahedron $K_{l} \in \mathcal{T}_{h}$, denoted as $\left.a_{i j}^{\text {pos }}\right|_{K_{l}}$, into $a_{i j}^{\text {pos }}$. Let $K_{l}$ have vertices $B_{i}, B_{j}, B_{s}, B_{t}$ (see Figure 4 ) and let $\varepsilon=\alpha_{i j}^{K_{l}}-\frac{\pi}{2}$. Let $\alpha_{i t}^{K_{l}}$ be the smallest angle among angles formed by the face $S_{s j t}$ and the other faces adjacent to it. Then by (24) and (14), in order to show that $I-C$ satisfies also (6b), it is enough to prove that

$$
\begin{aligned}
& \left.a_{i j}^{\text {pos }}\right|_{K_{l}}+\left.\frac{1}{2} a_{i t}^{\text {neg }}\right|_{K_{l}} \\
& \quad=\frac{\operatorname{meas}_{2} S_{i s t} \cdot \operatorname{meas}_{2} S_{j s t}}{9 \text { meas }_{3} K_{l}} \cdot \sin \varepsilon-\frac{\operatorname{meas}_{2} S_{i s j} \cdot \operatorname{meas}_{2} S_{t s j}}{9 \operatorname{meas}_{3} K_{l}} \cdot \frac{\cos \alpha_{i t}^{K_{l}}}{2} \leq 0 .
\end{aligned}
$$

This inequality holds, due to (19) and (22), if the value of $\varepsilon$ is such that

$$
\sin \varepsilon \leq \frac{C_{2}^{\prime}}{2 C_{2}^{\prime \prime}} \sin \theta_{1},
$$

which implies that $I-C$ satisfies (6b). From (17a) we see that condition (6c) holds for all entries of the matrix $I-C$, i.e., (15b) is valid.

Now we check whether (15c) holds, i.e., we prove that the negative entries of $D^{\text {neg }}$ are dominated by the corresponding positive terms of $C D^{\text {pos }}$ (see Remark 4).

By the definition of matrix $D$ the "worst" situation occurs if all contributions into some entry $a_{i j}, i \neq j$, are positive, see (17b). Let such an entry be $a_{12}$ for simplicity. Then $d_{12}^{\text {neg }}=-a_{12}^{\text {pos }} / a_{11}$. If we consider the corresponding entry $\left(C D^{\text {pos }}\right)_{12}$, then we have to check whether the following condition holds (cf. (16) and (17)):

$$
\left(C D^{\text {pos }}\right)_{12}+\left(D^{\text {neg }}\right)_{12}=\sum_{r=1}^{M} \frac{-a_{1, r+2}^{\text {neg }}}{2 a_{11}} \cdot \frac{-a_{r+2,2}^{\text {neg }}}{2 a_{r+2, r+2}}-\frac{a_{12}^{\text {pos }}}{a_{11}} \geq 0 .
$$

We have from (14) and the assumption that all contributions into $a_{12}$ are nonnegative that

$$
a_{12}^{\text {pos }}=\sum_{r=1}^{M}-\frac{\text { meas }_{2} S_{1, r+2, r+3} \cdot \text { meas }_{2} S_{2, r+2, r+3}}{9 \operatorname{meas}_{3} K_{r}} \cdot \cos \alpha_{12}^{K_{r}},
$$


where $\alpha_{12}^{K_{r}}$ stands for the internal angle in $K_{r}$ between faces $S_{1, r+2, r+3}$ and $S_{2, r+2, r+3}$. Thus, from (19), (20) and (27) we get that

$$
a_{12}^{\text {pos }} \leq \frac{M\left(C_{2}^{\prime \prime}\right)^{2}}{9 C_{3}^{\prime}} \cdot h \cdot \sin \varepsilon,
$$

where $\varepsilon=\max \left\{\alpha_{12}^{K_{r}}-\frac{\pi}{2}, r=1, \ldots, M\right\}$.

Consider now the sum from the left-hand side of the inequality in (26). From the basic assumption (BA) we conclude that

$$
\sum_{r=1}^{M} \frac{-a_{1, r+2}^{\mathrm{neg}}}{2 a_{11}} \cdot \frac{-a_{r+2,2}^{\mathrm{neg}}}{2 a_{r+2, r+2}} \geq \frac{\sin ^{2} \theta_{2} \cdot\left(C_{2}^{\prime}\right)^{4} \cdot h^{2}}{4 a_{11} \cdot a_{r+2, r+2} \cdot\left(C_{3}^{\prime \prime}\right)^{2}}
$$

Hence $\varepsilon$ is to be chosen so that

$$
\sin \varepsilon \leq \frac{9}{4 M} \cdot \frac{\left(C_{2}^{\prime}\right)^{4}}{\left(C_{2}^{\prime \prime}\right)^{2}} \cdot \frac{C_{3}^{\prime}}{\left(C_{3}^{\prime \prime}\right)^{2}} \cdot \frac{\sin ^{2} \theta_{2}}{a_{r+2, r+2}} \cdot h .
$$

Taking into account that (cf. (13), (19) and (20))

$$
a_{r+2, r+2} \leq \frac{\left(C_{2}^{\prime \prime}\right)^{2}}{9 C_{3}^{\prime}} h
$$

we observe that the value of $\varepsilon$ has to be such that

$$
\sin \varepsilon \leq \frac{81}{4 M_{\max }} \cdot \frac{\left(C_{2}^{\prime}\right)^{4}}{\left(C_{2}^{\prime \prime}\right)^{4}} \cdot \frac{\left(C_{3}^{\prime}\right)^{2}}{\left(C_{3}^{\prime \prime}\right)^{2}} \cdot \sin ^{2} \theta_{2} .
$$

The condition $(15 \mathrm{~d})$ can be trivially proved as in 13 .

Since, under the assumptions of the theorem, the value of $\varepsilon$ satisfies (25) and (28), then obtuse angles with values less or equal to $\frac{\pi}{2}+\varepsilon$ are allowed and the discrete maximum principle holds.

Consider now the situation when $B_{2} \in \partial \Omega$, but none of $B_{3}, B_{4}, \ldots, B_{M+2}$ belongs to $\partial \Omega$. In this case the entry $a_{12} \neq 0$ (but $a_{21}=0-$ cf. (11)), and the same arguments as before can be used, since inequality (26) does not involve any term of the form $a_{2 j}, j \neq 2$, that vanish now.

Further, let the function $g$ in (2) be constant and let, say, the face $B_{2} B_{3} B_{4}$ belong to the boundary. If we consider the entries $a_{12}, a_{13}, a_{14}$ being, in fact, entries of $Z$ from (11), then employing the arguments used to prove (24) and a simple observation from [13] p. 485], we may replace the positive entry $a_{12}$ with zero and change entries $a_{13}, a_{14}$ by nonpositive entries $a_{13}^{\prime}, a_{14}^{\prime}$, respectively, so that we get an equivalent system of equations with a new matrix already satisfying (6c).

\section{NUMERICAL EXPERIMENTS}

Consider problem (1)-(2) with $g=0$, where $\Omega$ is a parallelepiped whose shape is shown in Figure 5. Let us divide $\bar{\Omega}$ into $3 \times 3 \times 3=27$ smaller parallelepipeds which are congruent and similar to $\bar{\Omega}$.

In this way we obtain eight interior nodal points $B_{1}, \ldots, B_{8}$ and let their order correspond to Figure 6, i.e., $B_{1} \ldots B_{8}$ is one the 27 parallelepipeds, which is in the centre of $\Omega$. Assume that

$$
B_{4}=(0,0,0), \quad B_{5}=(8,0,0), \quad B_{3}=(-3,8,0), \quad B_{1}=(4,4,7) .
$$




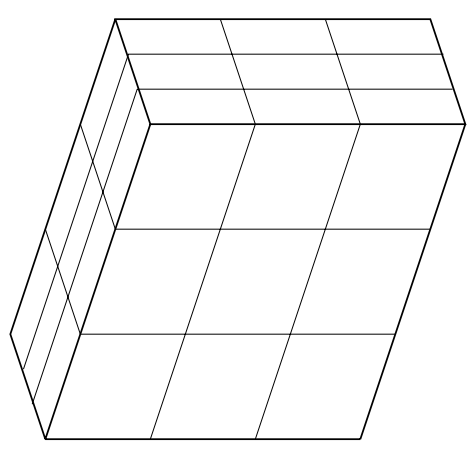

FiguRE 5 .

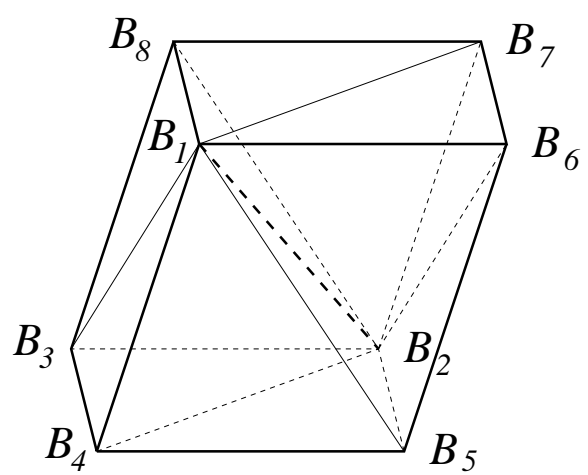

Figure 6.

Coordinates of the other vertices $B_{i}$ and the vertices of $\Omega$ can now be determined from the above by a simple calculation.

We decompose the interior parallelepiped $B_{1} \ldots B_{8}$ into 6 tetrahedra as sketched in Figure 6, and the other 26 parallelepipeds are decomposed in the same manner. Then the associated triangulation $\mathcal{T}_{h}$ of $\bar{\Omega}$ contains $6 \times 27=162$ tetrahedral elements having the same volume. Hence,

$$
C_{3}^{\prime}=C_{3}^{\prime \prime}
$$

in inequality (20). The triangulation $\mathcal{T}_{h}$ is, in fact, equivalent to decompositions studied in [9] and [12]. Note that each vertex $B_{i}$ belongs to 24 tetrahedra and each interior edge is surrounded by 4 or 6 tetrahedra. Therefore,

$$
M_{\max }=6 .
$$

Simple computer calculations now lead to

$$
C_{2}^{\prime}=31.532, \quad C_{2}^{\prime \prime}=37.125, \quad \theta_{1}=\theta_{2}=29.74^{\circ}
$$

(cf. (19) and (22)), see Remark 6. Then we find by Theorem 3, (29), (30) and (31) that

$$
\sin \varepsilon \leq \min (0.21070,0.43233)
$$

which yields the upper bound

$$
\varepsilon \leq 12.16^{\circ}
$$

The tetrahedron $B_{1} B_{2} B_{3} B_{4}$ has two obtuse angles: $92.78^{\circ}$ at the edge $B_{1} B_{3}$ and $100.30^{\circ}$ at the opposite edge $B_{2} B_{4}$. These edges are surrounded only by four tetrahedra from $\mathcal{T}_{h}$. Since the angle $100.30^{\circ}=90^{\circ}+10.30^{\circ}$ is at the same time the greatest angle in the whole triangulation, we see that (32) is valid, and thus the discrete maximum principle holds due to Theorem 3. 
The corresponding $8 \times 8$ stiffness matrix $A$ is monotone

$$
A=\left(\begin{array}{ccccccccc}
39.30 & & & & & & & \\
-5.54 & 39.30 & & & & & \text { sym. } & \\
0.97 & -5.79 & 39.30 & & & & & \\
-3.83 & -0.23 & -4.49 & 39.30 & & & & \\
-0.74 & -4.49 & 0 & -5.79 & 39.30 & & & \\
-5.79 & 0.97 & 0 & 0 & -3.83 & 39.30 & & \\
-0.23 & -3.83 & 0 & 0 & 0 & -4.49 & 39.30 & \\
-4.49 & -0.74 & -3.83 & 0 & 0 & 0 & -5.79 & 39.30
\end{array}\right)
$$

even though some of its nondiagonal entries are positive.

If an edge is surrounded only by four tetrahedra, then, of course, at least one angle is greater than or equal to $90^{\circ}$. For unstructured triangulations such an angle is greater than $90^{\circ}$, in general. The above example illustrates that angles which are even slighty greater than $100^{\circ}$ can still guarantee the validity of the strong discrete maximum principle. Other numerical experiments with similar parallelepipeds show that angles less than $100^{\circ}$ usually do not destroy the discrete maximum principle provided their faces have approximately the same area.

Finally, note that refinements of tetrahedral triangulations should be done with special care (see [11] and [19]). The latter reference contains an interesting example, where repeated refinement by midlines may produce a degenerate tetrahedra if the interior octahedron in Figure 1 is divided into 4 tetrahedra incorrectly.

\section{REFERENCES}

1. Bertolazzi, E., Discrete conservation and discrete maximum principle for elliptic PDEs, Math. Models Methods Appl. Sci. 8 (1998), 685-711. MR 99h:65185

2. Bramble, J. H., Hubbard, B. E., New monotone type approximations of elliptic problems, Math. Comp. 18 (1964), 349-367. MR 29:2982

3. Ciarlet, P. G., Discrete maximum principle for finite difference operators, Aequationes Math. 4 (1970), 338-352. MR 45:1404

4. Ciarlet, P. G., Raviart, P. A., Maximum principle and uniform convergence for the finite element method, Comput. Methods Appl. Mech. Engrg. 2 (1973), 17-31. MR 51:11992

5. Feistauer, M., Felcman, J., Rokyta, M., Vlášek, Z., Finite-element solution of flow problems with trailing conditions, J. Comput. Appl. Math. 44 (1992), 131-165. MR 94d:76052

6. Gilbarg, D., Trudinger, N. S., Elliptic partial differential equations of second order, SpringerVerlag, Berlin-New York, 1977. MR 57:13109

7. Golias, N. A., Tsiboukis, T. D., An approach to refining three-dimensional tetrahedral meshes based on Delaunay transformations, Internat. J. Numer. Methods Engrg. 37 (1994), 793-812. CMP 94:08

8. Höhn, W., Mittelmann, H. D., Some remarks on the discrete maximum-principle for finite elements of higher order, Computing 27 (1981), 145-154. MR 83a:65109

9. Křížek, M., An equilibrium finite element method in three-dimensional elasticity, Apl. Mat. 27 (1982), 46-75. MR 83b:73056

10. Kř́ízek, M., Qun, L., On diagonal dominance of stiffness matrices in 3D, East-West J. Numer. Math. 3 (1) (1995), 59-69. MR 96f:65153

11. Kř́ížek, M., Strouboulis, T., How to generate local refinements of unstructured tetrahedral meshes satisfying a regularity ball condition, Numer. Methods Partial Differential Equations, 13 (1997), 201-214. MR 98c:65160]

12. Ong, M. E. G., Uniform refinement of a tetrahedron, SIAM J. Sci. Comput. 15 (1994), 11341144. MR 95f:65213

13. Santos, V. R., On the strong maximum principle for some piecewise linear finite element approximate problems of nonpositive type, J. Fac. Sci. Univ. Tokyo Sect. IA Math. 29 (1982), 473-491. 
14. Stampacchia, G., Le problème de Dirichlet pour les équations elliptiques du second ordre á coefficients discontinus, Ann. Inst. Fourier (Grenoble) 15 (1968), 189-258. MR 33:404

15. Stoyan, G., On a maximum principle for matrices, and on conservation of monotonicity. With applications to discretization methods., Z. Angew. Math. Mech. 62 (1982), 375-381. MR 83k:65067

16. Stoyan, G., On maximum principles for monotone matrices, Linear Algebra Appl. 78 (1986), 147-161. MR 87k:65032

17. Strang, G., Fix, G. J., An analysis of the finite element method, Prentice Hall, Englewood Cliffs, N. J., 1973. MR 56:1747

18. Varga, R., Matrix iterative analysis, Prentice Hall, New Jersey, 1962. MR 28:1725

19. Zhang, S., Successive subdivisions of tetrahedra and multigrid methods on tetrahedral meshes, Houston J. Math. 21 (1995), 541-556. MR 96f:65183

University of Jyväskylä, Department of Mathematical Information Technology, P.O. Box 35, FIN-40351 JyvÄSKYLÄ, Finland

E-mail address: korotov@mit.jyu.fi

Mathematical Institute, Academy of Sciences, Žitná 25, CZ-11567 Prague 1, Czech REPUBLIC

E-mail address: krizek@math.cas.cz

University of Jyväskylä, Department of Mathematical Information Technology, P.O. Box 35, FIN-40351 Jyvëskylë, Finland

E-mail address: pn@mit.jyu.fi 